\title{
Histological characterisation of the skin of the Paraechinus hypomelas, Brandt, 1836 (Erinaceidae: Eulipotyphla)
}

\author{
M. Akbari Bazm¹, N. Goodarzi², M.M.A. Abumandour ${ }^{3}$, L. Naseri' ${ }^{1}$, M. Hosseinipour ${ }^{4}$ \\ ${ }^{1}$ Department of Anatomical Sciences, Medical School, Kermanshah University of Medical Sciences, Kermanshah, Iran \\ 2Department of Basic Sciences and Pathobiology, Faculty of Veterinary Medicine, Razi University, Kermanshah, Iran \\ ${ }^{3}$ Department of Anatomy and Embryology, Faculty of Veterinary Medicine, Alexandria University, Alexandria, Egypt \\ ${ }^{4}$ DVM student, Faculty of Veterinary Medicine, Razi University, Kermanshah, Iran
}

[Received: 9 March 2019; Accepted: 19 May 2019]

Background: The current study represents the first description of the histological characterisations of the normal skin of Brandt's hedgehog (paraechinus hypomelas).

Materials and methods: Skin samples were collected from abdomen, back, nostril and cloacal regions.

Results: The skin consisted of 3 layers including epidermis, dermis and hypodermis. The epidermis was covered by a layer of keratinised squamous epithelium mainly in the back region, but the skin keratinisation was present with a little amount or may was absent in other regions. Histologically, the total thickness of skin was maximum on the back and minimum on the cloacal regions. The epidermis consisted of 4 layers and stratum lucidum was absent in all regions. Beneath the epidermis, the dermis layer was constituted of dense connective tissue in which the hair follicles, sweat glands, sebaceous glands, arrector pilli muscles and blood vessels were present. The sweat and sebaceous glands were more populated in the nostril region. The hair follicles were located in the epidermal and dermal regions. Vibrissae were only in the nostrils region and characterised from other hairs by their large and well innervated hair follicle which was surrounded by the blood sinus.

Conclusions: The present findings show that in Brandt's hedgehog (paraechinus hypomelas) the back and cloacal regions have thickest and thinnest skin respectively as compared to the nostril and abdominal regions. In addition, sebaceous and sweat glands were mainly populated in the nostril region. (Folia Morphol 2020; 79, 2: 280-287)

Key words: hedgehog, histology, skin, sweat gland, Paraechinus hypomelas

\section{INTRODUCTION}

The hedgehog is described as a spiny mammal species. Generally, there are 17 species of the hedgehog which are classified in 5 genera and distributed in certain parts of Asia, Africa, and Europe. The Brandt's hedgehog (paraechinus hypomelas) species belongs to the Eulipotyphla order, Erinaceidae family,
Erinaceinae subfamily, and Paraechinus genus [21]. The Brandt's hedgehog is mainly nocturnal species and lives in the desert regions of the Middle East and Middle Asia [21]. The animal integument gives the mechanical protection of the underlying tissue and acts as a barrier against external infection and the different environmental conditions [10]. In addition to 
Table 1. Thicknesses $(\mu \mathrm{m})$ of skin layers in Brandt's hedgehog $(n=5)$

\begin{tabular}{|c|c|c|c|c|c|}
\hline \multirow[t]{2}{*}{ Body regions } & \multicolumn{2}{|c|}{ Epidermal thickness } & \multirow[t]{2}{*}{ Dermal thickness } & \multirow[t]{2}{*}{ Hypoderm } & \multirow[t]{2}{*}{ Total thickness } \\
\hline & Corneal & Cellular & & & \\
\hline Abdomen & $17.6 \pm 6.9^{a}$ & $30.9 \pm 10.9^{a}$ & $440.9 \pm 45.8^{\mathrm{a}}$ & $352.8 \pm 60.6^{a}$ & $560.06 \pm 68.44^{a}$ \\
\hline Back & $45.6 \pm 10.2^{b}$ & $20.6 \pm 7.9^{\mathrm{ab}}$ & $2041.9 \pm 190.9^{b}$ & $1150.9 \pm 170.9^{b}$ & $2226.60 \pm 140.60^{b}$ \\
\hline Cloak & - & $17.4 \pm 8.7^{b}$ & $468.9 \pm 61.8^{a}$ & $249.9 \pm 50.8^{\mathrm{a}}$ & $520.32 \pm 45.22^{\mathrm{a}}$ \\
\hline Nostril & $30.8 \pm 8.1^{c}$ & $140.3 \pm 20.6^{c}$ & $413.6 \pm 45.9^{a}$ & $125.9 \pm 25.4^{c}$ & $620.14 \pm 14.26^{a}$ \\
\hline
\end{tabular}

Values are expressed as mean \pm standard error. Different superscripts in the same column indicate significant difference between regions $(p<0.05)$.

the biological and immunological values, the skin has an important economic importance in the industry as a raw material which introduce in many important industry [13]. The structure of the skin can be influenced by several factors including living environment (temperature, humidity, etc.) and nutritional habits. Therefore, thickness or strength (collagen content) of skin layers is species-specific. In addition, the skin of different regions can vary regarding to the thickness of layers, type of glands, and presence or density of hair follicles [6]. For instance, Kietzmann et al. [7] revealed that rat's tail has histologically different epidermis in comparison to other parts of the body. Furthermore, gender and age in a given species can influence the skin histology. It has been shown that, in rat the thickness of dermis increases with age, so that it reaches up to 5 times in 1 year [19]. There are some morphological descriptions of the skin of other mammals' species such as ferret, camel, and cat [1, 9, 23]. However, there is a scanty available data about the normal properties of the skin of hedgehog, especially the Brandt's hedgehog (Paraechinus hypomelas) species. So, the current study was conducted to explain the histological structure of the skin of Brandt's hedgehog captured from Kermanshah city. Finally, the obtained results were compared with those reported in other mammalian species.

\section{MATERIALS AND METHODS}

\section{Samples collection}

Five male animals were included in the present study. The dead animals were found around the rural regions of Kermanshah city and transferred to dissection room of Faculty of Veterinary Medicine. The exact time of death was unclear; however, based on the appearance of the viscera, it seemed less than $2 \mathrm{~h}$ past from their death. The present study performed according to the guideline of Committee on Animal Welfare and Ethics of Razi University.

\section{Microscopic examination}

Small specimens $\left(1 \mathrm{~cm}^{3} \times 1 \mathrm{~cm}^{3}\right)$ of the skin from four different regions of the body including nostril, back, abdomen and cloacae were sampled. The skin samples were fixed in $10 \%$ neutral buffered formaldehyde for 10 days. For histological observation, skin samples were routinely processed and embedded in paraffin. The histological sections with 5-7 $\mu \mathrm{m}$ thickness were prepared using Leica rotatory microtome (RM 20352035; Leica Microsystems, Wetzlar, Germany) and mounted on glass slides. Paraffin sections were used for conventional staining (haematoxylin and eosin), in addition to Masson trichrome and periodic acid Schiff (PAS) staining methods. Finally, the sections were analysed and epidermal (corneal and cellular), dermal and hypodermal thickness were measured using a light microscope (Nikon YS100, Japan) equipped with a KEcamera (KEcam Technologies, Lekki Lagos, Nigeria) and Top view software (Version 3.7). The images were processed using Adobe Photoshop CC (Adobe system, San Jose, CA, USA). The density of sebaceous and sweat glands were calculated in different regions of the Brandt's hedgehog using a lattice line graticule $(5 \times 5)$.

\section{Data analysis}

Data were analysed using the one-way ANOVA in SPSS/PC software (Version 23) followed by Tukey's post-hoc test. All values were expressed as mean \pm standard error.

\section{RESULTS}

\section{Histometrical study}

The thickness of skin layers in four different examined regions were described in the Table 1. The mean values of total dermal thickness in abdomen, back, cloak and nostril regions were $440.9 \pm 45.8 \mu \mathrm{m}$, $2041.9 \pm 190.9 \mu \mathrm{m}, 468.9 \pm 61.8 \mu \mathrm{m}$ and $413.6 \pm 45.9 \mu \mathrm{m}$, respectively. The epidermis of the back region had thickest keratinised layer $(45.6 \pm 10.2 \mu \mathrm{m})$, 
Table 2. The density of the sebaceous and sweat gland in the Brandt's hedgehog skin $(n=5)$

\begin{tabular}{lcc}
\hline Body regions & Sebaceous gland $/ \mathrm{mm}^{2}$ & Sweat gland $/ \mathrm{mm}^{2}$ \\
\hline Abdomen & $3.41 \pm 0.68^{\mathrm{a}}$ & $3.32 \pm 0.24^{\mathrm{a}}$ \\
Back & $3.52 \pm 0.32^{\mathrm{a}}$ & $4.23 \pm 0.81^{\mathrm{a}}$ \\
Cloak & - & $3.25 \pm 0.15^{\mathrm{a}}$ \\
Nostril & $8.61 \pm 0.95^{\mathrm{b}}$ & $9.51 \pm 1.34^{\mathrm{b}}$ \\
\hline
\end{tabular}

Values are expressed as mean \pm standard error. Different superscripts in the same column indicate significant difference between regions $(p<0.05)$.

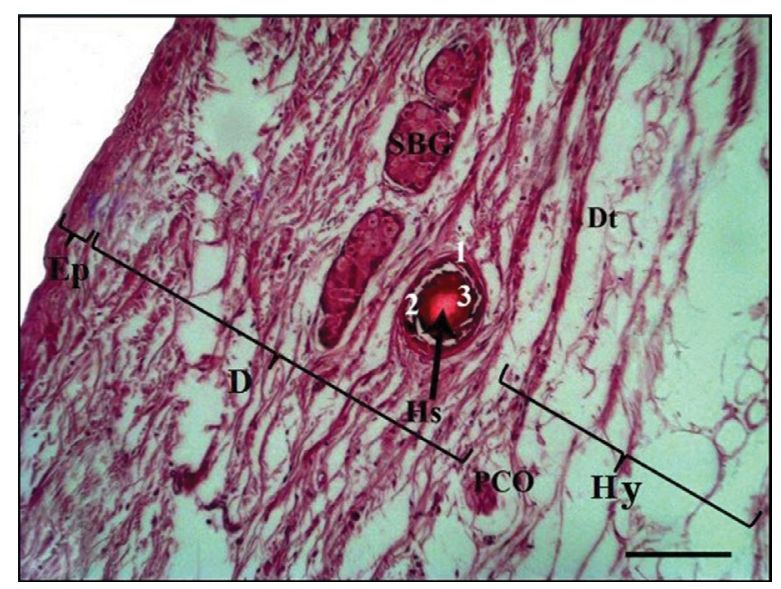

Figure 1. Light photomicrograph of the normal skin of the abdomen region in the Brandt's hedgehog; SC — stratum corneum; SS — stratum spinosum; Stb - stratum basale; Ep — epidermis; $\mathrm{D}$ - dermis; Hy - hypodermis; SBG — sebaceous gland; Hs - hair shaft; Dt —adipose tissue; PCO — Pacinian corpuscle; 1 - external root sheath; 2 - internal root sheath; 3 - hair cortex; haematoxylin and eosin, bar $=100 \mu \mathrm{m}, \times 40$.

while the thinnest keratinised layer was seen in the abdominal region $(17.6 \pm 6.9 \mu \mathrm{m})$. The keratinised layer was absent in the cloacal region. The thickest and thinnest cellular parts of the epidermis were measured in the nostril and cloacal regions, respectively $(140.3 \pm 20.6$ and $17.4 \pm 8.7 \mu \mathrm{m})$. The highest value for the hypodermal thickness was $1150.9 \pm 170.9 \mu \mathrm{m}$ in the back and the lowest value was $125.9 \pm 25.4 \mu \mathrm{m}$ in the nostril region (Table 1).

The density of the sebaceous and sweat glands in the nostril region $(8.61 \pm 0.95$ and $9.51 \pm 1.34$ unit $/ \mathrm{mm}^{2}$, respectively) was significantly higher than other examined regions. The difference between back, abdominal and cloacal regions was not statistically significant (Table 2).

\section{Histological study}

The micromorphological structure of the Brandt's hedgehog (paraechinus hypomelas) skin consisted of 3 layers namely epidermis (external layer), dermis

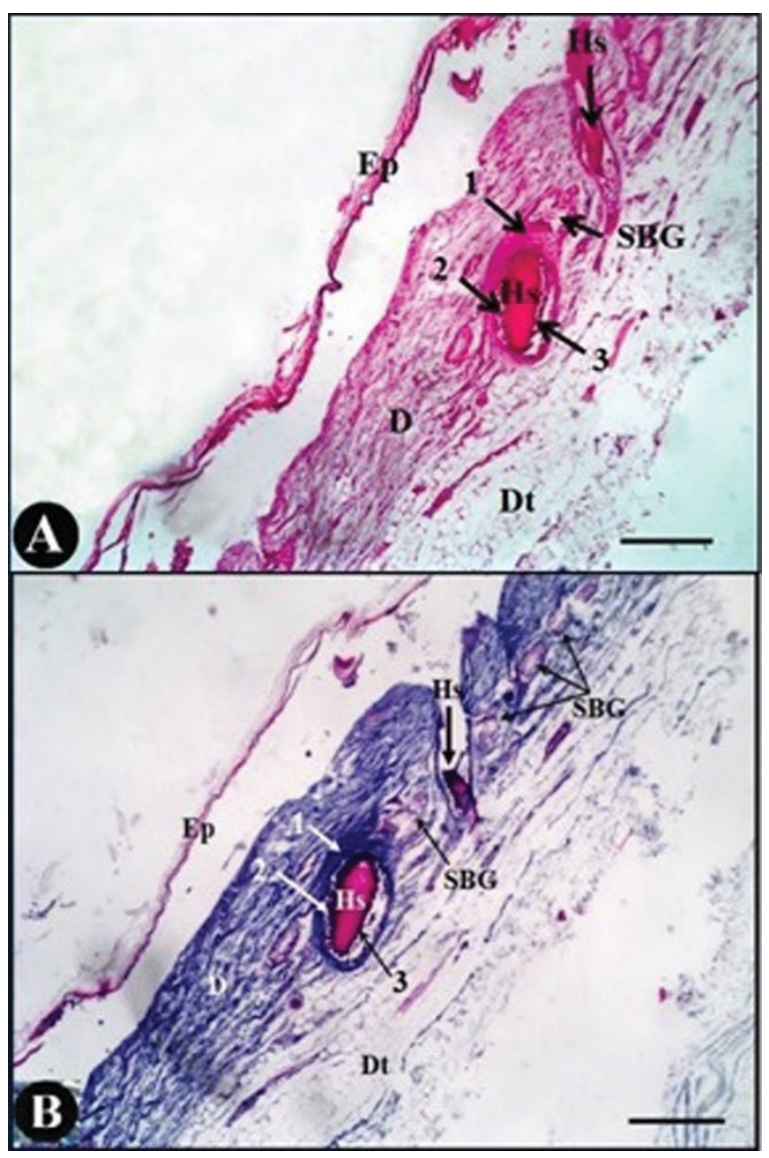

Figure 2. Light photomicrograph of the normal skin of the abdomen region in the Brandt's hedgehog; haematoxylin and eosin staining (A) and Masson's trichrome staining (B); Ep - epidermis; D dermis; SBG — sebaceous gland; Dt - adipose tissue; Hs - hair shaft; 1 - external root sheath; 2 - internal root sheath; 3 - hair cortex; bar $=100 \mu \mathrm{m}, \times 40$.

(middle layer) and hypodermis (internal layer) (Figs. 1; 2; 3B and 5). The epidermis was covered externally by a thick layer of keratinised material in the back region (Fig. 3B), but a little amount of cornification was present over the abdominal epidermis (Figs. 1; 2).

The cellular part of the epidermis consisted of four layers especially in the nostril region and was arranged from outside to inside as the following; stratum corneum, stratum granulosum, stratum spinosum, and stratum basale (Figs. 5A; 6B; 7B). These layers were not distinguishable clearly in the abdominal, back, and cloacal regions (Figs. 1; 2; 3B; 8). The excretory ducts of sweat glands were seen in cellular epidermis of the nostril region (Figs. 5A; 6B; 7B).

The dermis constituted of dense irregular connective tissue especially in the back and nostril regions with highly compact collagen bundles (Figs. 3B; 5). But it was more loose in the abdominal and cloacal regions (Figs. $1 ; 8$ ). There was many important struc- 


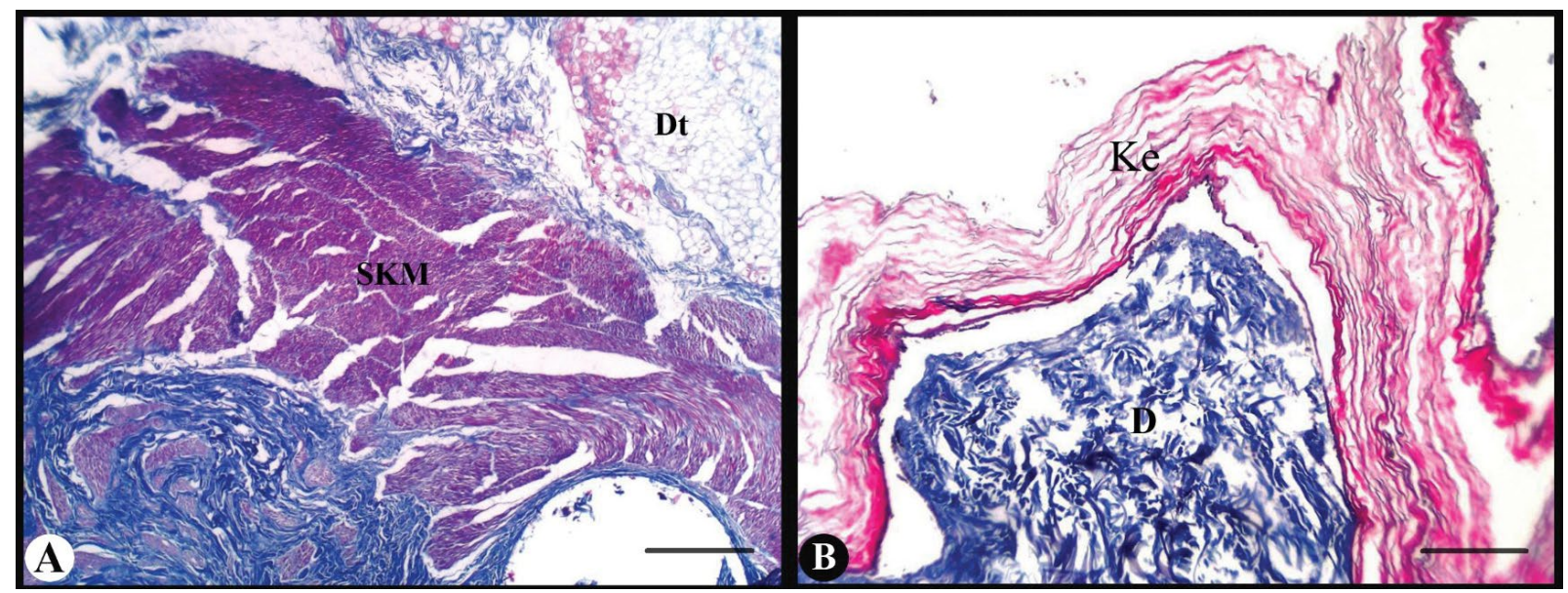

Figure 3. Light photomicrograph of the normal skin of the back region in the Brandt's hedgehog shows mass of skeletal muscle (SKM), adipose tissue (Dt) (A), and shows thick keratinised layer of epidermis (Ke), and dermis (D) (B); Masson's trichrome staining, bar $=100 \mu \mathrm{m}, \times 100$.

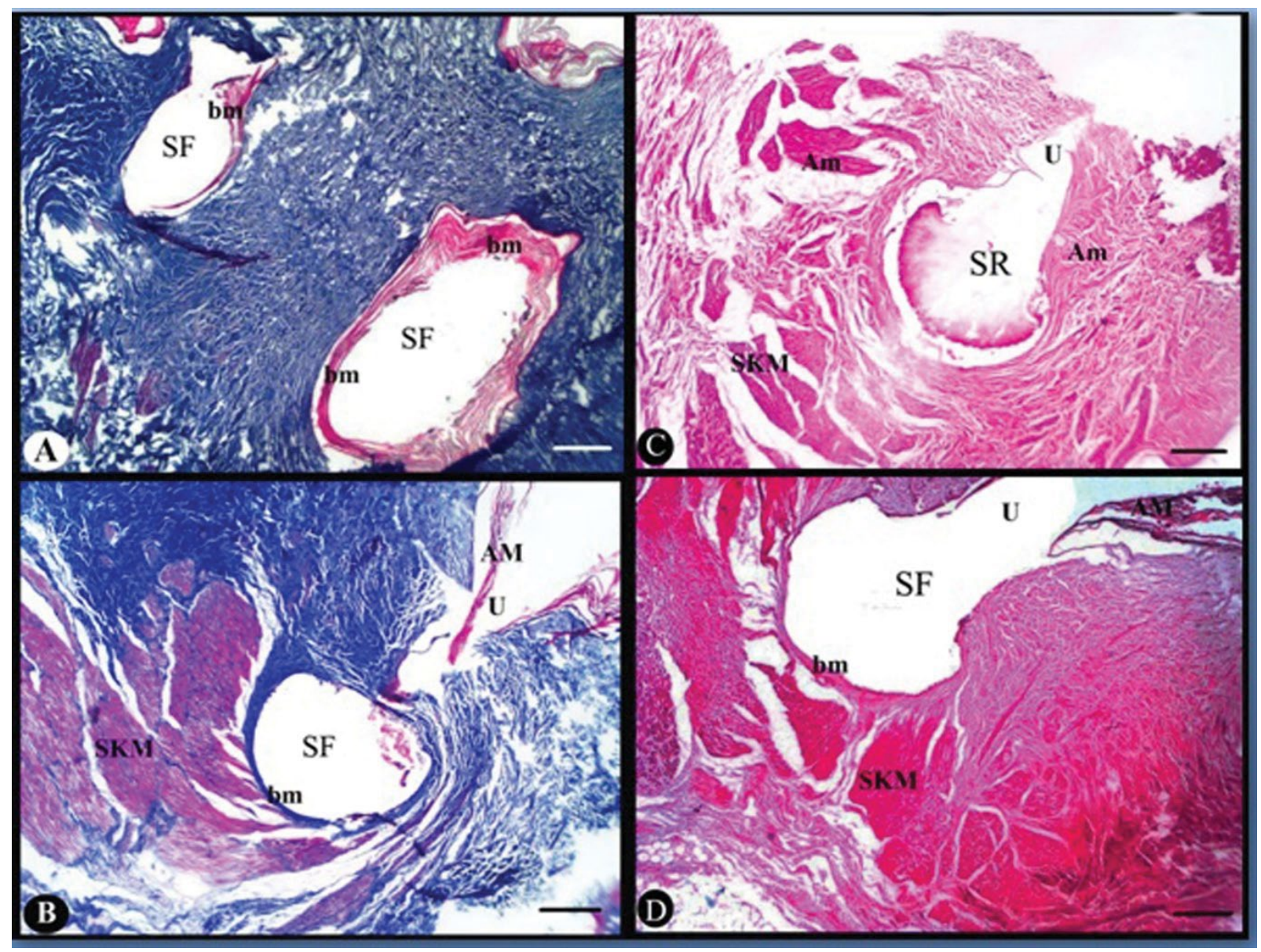

Figure 4. Light photomicrograph of the normal skin of the back region in the Brandt's hedgehog. A, B. Masson's trichrome staining; C, D. Haematoxylin and eosin staining. Bright pink basement membrane $(\mathrm{bm})$ at the dermal-epidermal junction around the spine follicle (SF) in cross section; $\mathrm{SR}$ - spine root; SKM — skeletal muscle; Am — arrector pilli muscle; U — upper portion of hair shaft (infundibulum); bar $=50 \mu \mathrm{m}, \times 100$.

tures including sweat gland, hair follicle, and nerve ending in all examined region, sebaceous gland in all examined regions except in the cloacal region, spine follicles in the back region (Fig. 4), and vibrissae (tactile hair) in the nostril region (Fig. 6A). The interdigitation between the dermis and epidermis as dermal 


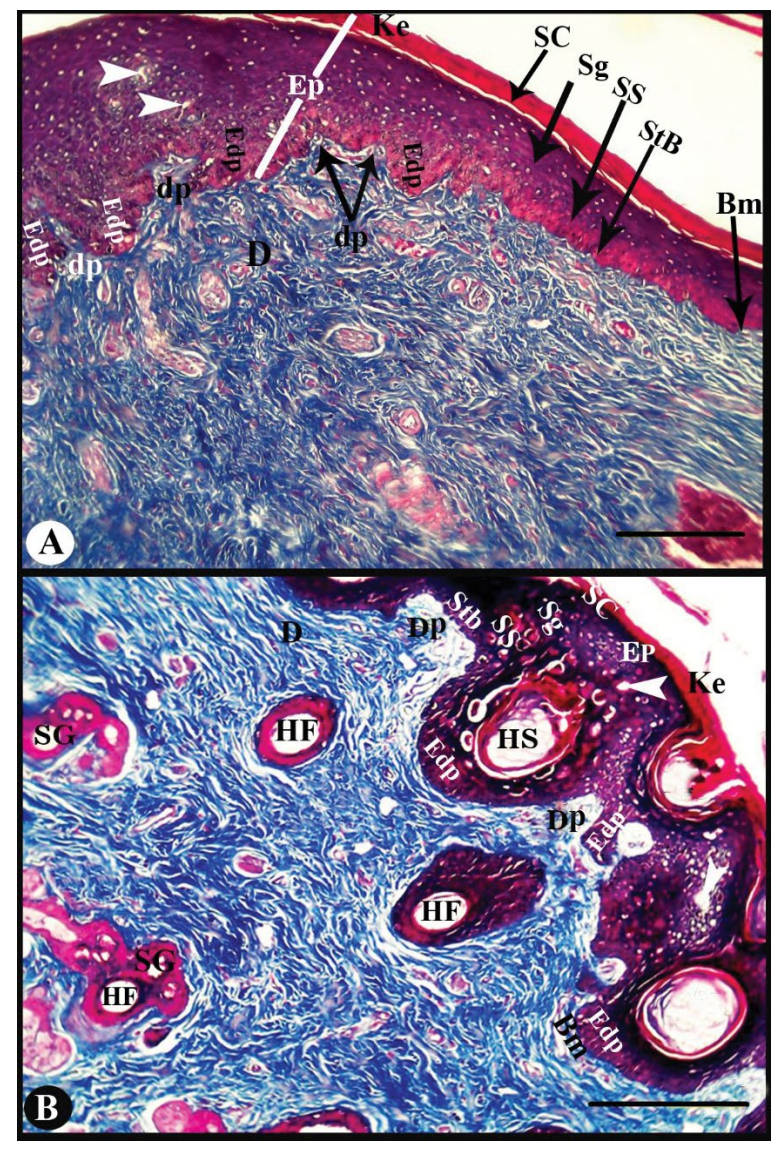

Figure 5. Light photomicrograph of the normal skin of the nostril region in the Brandt's hedgehog without hair follicles (A) and with hair follicles (B); Ke - keratinised material; SC — stratum corneum; SS - stratum spinosum; $\mathrm{Sg}$ - stratum granulosum; $\mathrm{StB}$ - stratum basale; Ep — epidermis; D — dermis; $\mathrm{Bm}$ — basement membrane; Edp - epidermal peg; Dp — dermal papillae; HF — hair follicle; SG - sweat gland; white arrowheads - excretory ducts of the sweat glands; Masson's trichrome staining; bar $=100 \mu \mathrm{m}, \times 100$.

papillae and epidermal peg was more prominent in the nostril region (Figs. 5-7).

The hypodermis consisted of loose connective tissue and adipose tissue (Figs. $1 ; 2 ; 3 \mathrm{~A} ; 8$ ). In the back region, this layer contained a huge amount of striated muscle fibres which surrounded spine follicles (Figs. 3A; 4B, D).

\section{DISCUSSION}

The present work attempted to describe histological properties of the skin in Brandt's hedgehog (Paraechinus hypomelas). Previous studies have shown that the thickness of different layers of skin (dermis and epidermis) and its structures depends on various factors such as age, gender, ethnicity, living environment, nutrition, etc. [12]. Micromorphologically, the skin of the Brandt's hedgehog consisted of

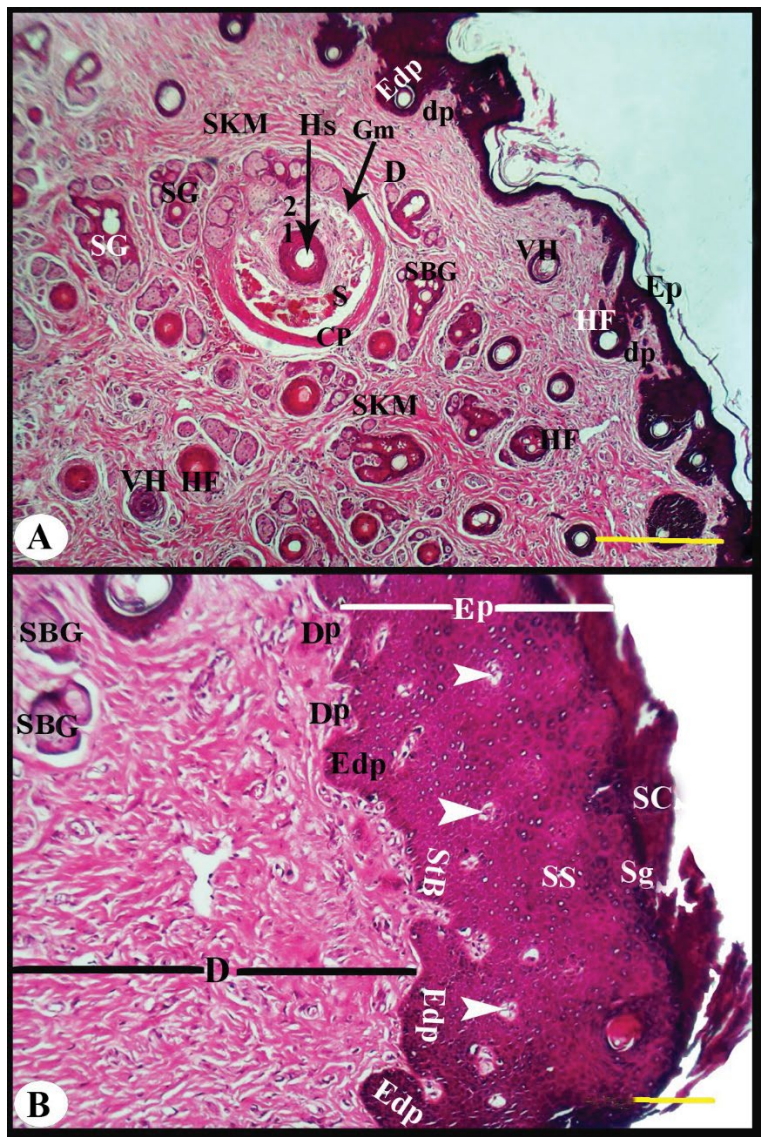

Figure 6. Light photomicrographs of the normal skin adjacent to the nostril with thin epithelium containing vibrissae hair $(\mathbf{A})$ and with thick epithelium (B) in the Brandt's hedgehog; $\mathrm{Ke}$ - keratinised material; Ep — epithelium; D — dermis; SC — stratum corneum; $\mathrm{SS}$ - stratum spinosum; $\mathrm{Sg}$ - stratum granulosum; $\mathrm{StB}$ - stratum basale; Edp — epidermal peg; Dp — dermal papillae; $\mathrm{VH}$ - vibrissae hair follicle; HF — hair follicle; Hs - hair shaft; SG — sweat gland; SBG — sebaceous gland; SKM — skeletal muscle: white arrowheads - excretory ducts of the sweat glands; $C P$ - capsule; $\mathrm{S}$ - sinus; 1 - inner root sheath; 2 - outer root sheath; $\mathrm{Gm}$ glassy membrane; haematoxylin and eosin; bar $=200 \mu \mathrm{m}, \times 100$.

3 layers namely epidermis, dermis and hypodermis as described in many animal species [20]. While, in the Bakerwali goat, and cattle the skin has been described that consisted of 2 layers; epidermis and dermis $[14,17]$.

Several studies have shown that the thickness of epidermis depends on a variety of factors, including the position of skin in the body, age, gender, pigmentation, and the amount of blood supply. One study on human skin showed that the thickest epidermal layer $(81.5 \mu \mathrm{m})$ was in the buttock area and the thinnest $(56.6 \mu \mathrm{m})$ in the upper back area of the upper limb. The highest thickness of stratum corneum of the epidermis was related to the upper dorsal area of the body $(18.3 \mu \mathrm{m})$ and the lowest thickness $(11 \mu \mathrm{m})$ was 


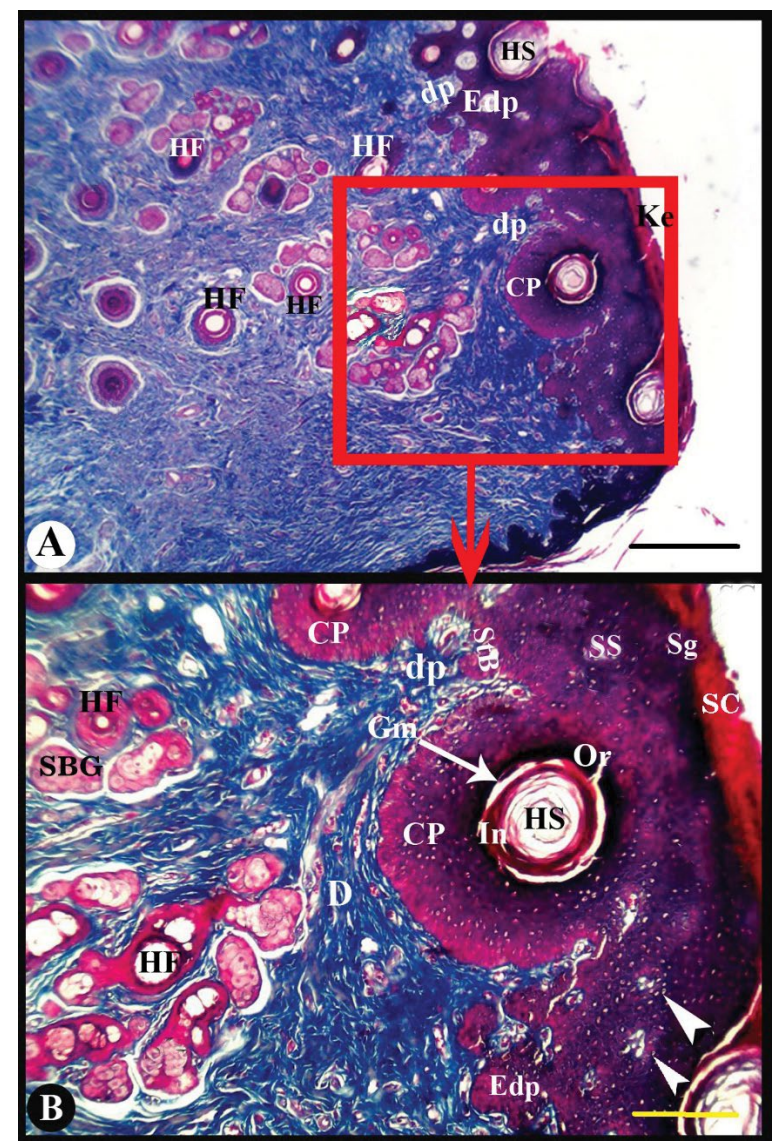

Figure 7. Light photomicrograph of the normal skin of the nostril region in the Brandt's hedgehog $(\mathbf{A})$. Red rectangle shown in higher magnification (B); Ke — keratinised material; SC — stratum corneum; SS - stratum spinosum; Sg — stratum granulosum; $\mathrm{StB}$ - stratum basale; D - dermis; HF — hair follicle; HS — hair shaft; CP — capsule; Edp — epidermal peg; dp — dermal papillae; SGB — sebaceous gland; In — inner root sheath; $\mathrm{Or}$ - outer root sheath; white arrowheads - excretory ducts of the sweat glands; Masson's trichrome staining; scale bar $200 \mu \mathrm{m}$; the red rectangle shown in panel $B$ at higher magnification; bar $=100 \mu \mathrm{m}, \times 100$.

in the shoulder. The corneum layer was less related to age and gender changes than the cell layer [15]. The thickest skin with thickest corneum layer was observed in the back area of the hedgehog, while the cloacal area had the thinnest skin. This difference may be due to the water retention, presence of spines and absorption of light by the back region. Theerawatanasirikul et al. [18] examined the thickness of epidermis in different parts of the body according to the age and gender of the dog. They found that the epidermis thickness was $35 \mathrm{~mm}$ in male dogs and $36.10 \mathrm{~mm}$ in female ones. Thickness of the epidermis was $33.2 \mathrm{~mm}$ in dogs less than 3 years old and $37 \mathrm{~mm}$ in dogs older than 7 years. This difference may be due to the protective role of the skin to prevent water withdrawal [18].

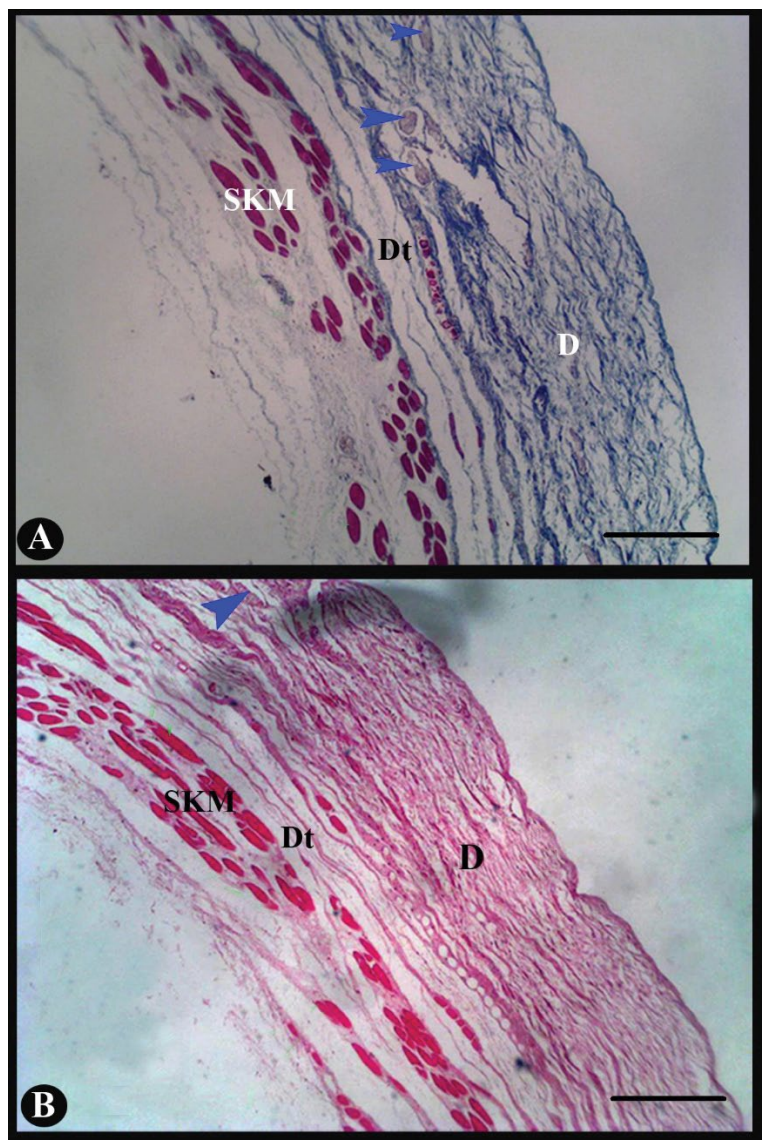

Figure 8. Light photomicrograph of the normal skin of the cloacal region in the Brandt's hedgehog with Masson's trichrome staining (A) and haematoxylin and eosin staining (B); D - dermis; SKM skeletal muscle in hypodermis, blue head arrows refer to cross section of nerve; bar $=100 \mu \mathrm{m}, \times 40$.

Corcuff et al. [3] calculated the thickness of human epidermis using a confocal microscope. The thickness of granulosum layer was $12-15 \mu \mathrm{m}$, and the stratum spinosum was reported to be $9-12 \mu \mathrm{m}$. In addition, the thickness of stratum basale was 6-8 $\mu \mathrm{m}$, and overall thickness of epidermis was $36 \mu \mathrm{m}$. Sumena et al. [16] reported the thickness of skin in the muzzle, neck, abdomen, and ankles of Yorkshire white pig to be 4, 3.7, 3.68, and $4.15 \mathrm{~mm}$, respectively. The hypoderm was not included in thickness. In studying the effect of age on human epidermal changes, it has been revealed that the thickness of the epidermis was reduced from $49 \mu \mathrm{m}$ in women and $63 \mu \mathrm{m}$ in men aged 20 to 30 years to $33 \mu \mathrm{m}$ in women and $33 \mu \mathrm{m}$ in men aged 70 to 80 years. However, the thickness of dermis was increased from $212 \mu \mathrm{m}$ in women, and $256 \mu \mathrm{m}$ in men aged 20-30 years to $251 \mu \mathrm{m}$ in women and $257 \mu \mathrm{m}$ in men aged 70 to 80 years [2]. The epidermis of the Brandt's hedgehog consisted mainly of 
4 layers which arranged from outside to inside as the following: stratum corneum, stratum granulosum, stratum spinosum, and stratum basale as described by some authors $[14,20]$. Furthermore, at the junction between the epidermis and dermis, epidermal pegs were interdigitated with corresponding dermal papillae especially in the nostril region. These results were noted also by Razvi et al. [14].

Thickness of various layers of skin depends on the animals breed. In the Mertolenga race of Portuguese native cattle, the thickness of corneum layer of skin is $2.6 \mu \mathrm{m}$, while in the Friesian race it reaches $8 \mu \mathrm{m}$. Thickness of the cell layers Friesian race is $38 \mu \mathrm{m}$, but it reaches $49 \mu \mathrm{m}$ in the Limousin race as well [11]. Dorsal dermis contains mainly thorns that cover the surface of the skin. These thorns are loose in the lower layers of the dermis. This feature improves the movement of the animal in small areas. The hypodermic layer is very thick in the back area and extends even to the epidermal folds. Below the hypodermis, there is a thick muscular layer called panniculus carnosus. Zaki [22] found that thickness of epidermis reduced by age in back, earlobe, and forefoot of albino rat aged 1,2 and 27 months. The thickness of epidermis in these areas at 6 months of age, as the puberty age, was reported to be $12,9.4,13.3$, and $48 \mu \mathrm{m}$, respectively. In addition, the thickness of pilosebaceus was $20,21.9,30.6$ and $11.3 \mu \mathrm{m}$ at this age [22].

The thickness of the stratum corneum was reported 93,14 and $16 \mu \mathrm{m}$, respectively in cow, human and rat lip. Thickness of stratum spinosum was 527 , 44 and $91 \mu \mathrm{m}$, stratum basale 54, 7 and $11 \mu \mathrm{m}$, and the granulosum layer was reported only $7 \mu \mathrm{m}$ in rats. These differences may be caused by the role of lips in feeding, preserving water and reducing body temperature in different animals [8]. The highest amount of sebaceous glands was seen in the muzzle area (nostril) of the Brandt's hedgehog, while there was no sebaceous gland in the cloak region. Most of the sweat glandular acini were found in the muzzle section and the least sweat glands were seen in the abdominal and cloacal regions. In native cattle of Bangladesh, the highest number of sebaceous glands was reported in the foot area $\left(2.08 \mathrm{~mm}^{2}\right)$ and the lowest number in the shoulder area $\left(0.46 \mathrm{~mm}^{2}\right)$. Furthermore, the number of glands in females was significantly more than males [4]. The vibrissae hairs named as whiskers and described as specific tactile sense hair found in most mammalian species except humans and were located exclusively in the nostril region of the Brandt's hedgehog. They were also observed in ferret, dog, and rat $[5,9,20]$.

The presence of elastic fibres in the back of the Brandt's hedgehog was noticeable in comparison to other parts, while in the cloacal region, these strands were observed rarely. Most blood vessels and nerves were seen in the muzzle area which can be served for sense of touch and concentration of mucosal secretions. There were a lot of blood vessels around the follicles of the spines in the back area. Pacinian corpuscles contribute to the sense of tension and compression of the skin in the reticular dermis of the abdomen. Skeletal muscles of skin were placed in the vicinity of the spines in the back region and contributed to their movement. The hair follicles were numerous in the muzzle area and extended to the reticular portion of dermis. They used in breathing, sense of smell and tactile sensation of the Brandt's hedgehog. The mucosal glands and their pores were seen in the muzzle and play a role in keeping this area wet. A layer of fat was observed in the abdominal and cloacal regions. This layer was not well developed in the back and the muzzle. Because of hibernation in some animals, the thickness of skin in different areas of some animals such as the hedgehog, and distribution of sebaceous and sweat glands varied from other animals, because there is a need for water retention and avoidance of the energy loss through heat. Sebaceous glands are dependent on the animal's living environment, gender, and season. The foods affect the level of sex hormones which play a role in the number and distribution of these glands. Sebaceous glands, which are altered by age in animals, are $8.6 \mu \mathrm{m}$ in lactation, $4.2 \mu \mathrm{m}$ in puberty and $3.2 \mu \mathrm{m}$ in old ages [23].

\section{CONCLUSIONS}

Overall, the present findings show that in Brandt's hedgehog (paraechinus hypomelas) the back and cloacal regions have thickest and thinnest skin respectively as compared to the nostril and abdominal regions. In addition, sebaceous and sweat glands were mainly populated in the nostril region of the body.

\section{REFERENCES}

1. Ansari-Renani HR, Salehi M, Ebadi Z, et al. Identification of hair follicle characteristics and activity of one and two humped camels. Small Ruminant Research. 2010; 90(1-3): 64-70, doi: 10.1016/j.smallrumres.2010.01.004.

2. Branchet $M C$, Boisnic $S$, Frances $C$, et al. Skin thickness changes in normal aging skin. Gerontology. 1990; 36(1): 2835, doi: 10.1159/000213172, indexed in Pubmed: 2384222. 
3. Corcuff $P$, Bertrand C, Leveque JL. Morphometry of human epidermis in vivo by real-time confocal microscopy. Arch Dermatol Res. 1993; 285(8): 475-481, doi: 10.1007/ bf00376820, indexed in Pubmed: 8274036.

4. Ershad Hossain MD, Uddin M, Kumar SS. Histomorphometrical characterization of skin of native cattle (bos indicus) in bangladesh. Am J Med Biol Res. 2016; 4: 53-65, doi: 10.12691/ajmbr-4-3-3.

5. Hartschuh W, Weihe E, Yanaihara N, et al. Immunohistochemical localization of vasoactive intestinal polypeptide (VIP) in Merkel cells of various mammals: evidence for a neuromodulator function of the Merkel cell. J Invest Dermatol. 1983; 81(4): 361-364, doi: 10.1111/1523-1747. ep12519966, indexed in Pubmed: 6137503.

6. Hildebrand M. Analysis of vertebrate structure. 3rd edition. John Wilet and Sons Inc. 1988.

7. Kietzmann $M$, Lubach $D$, Heeren $H J$. The mouse epidermis as a model in skin pharmacology: influence of age and sex on epidermal metabolic reactions and their circadian rhythms. Lab Anim. 1990; 24(4): 321-327, doi: 10.1258 /002367790780865921, indexed in Pubmed: 2270041.

8. Lanfranchi HE, de Rey BM. Comparative morphometric analysis of vermilion border epithelium and lip epidermis. Cells Tissues Organs. 1978; 101(2): 187-191, doi: 10.1159/000144965.

9. Martin AL, Irizarry-Rovira AR, Bevier DE, et al. Histology of ferret skin: preweaning to adulthood. Vet Dermatol. 2007; 18(6): 401-411, doi: 10.1111/j.1365-3164.2007.00627.x, indexed in Pubmed: 17991157.

10. Montage W. The structure and function of skin. 2nd ed. Academic Press, New York (NY) 1960.

11. Morais $\mathrm{P}$, Capela F, Silva F, et al. Measurements on the epidermis and sweat glands of four bovine breeds. Revista Portuguesa de Zootecnia. 2001; 8: 96-100.

12. Need AG, Morris HA, Horowitz M, et al. Effects of skin thickness, age, body fat, and sunlight on serum 25-hydroxyvitamin D. Am J Clin Nutr. 1993; 58(6): 882-885, doi: 10.1093/ajcn/58.6.882, indexed in Pubmed: 8249872.
13. Özfiliz N, Balikcir M, Erdost $H$, et al. Histological and morphometric features of the skin of native and hybrid (F_1) sheep. Turk J Vet Anim Sci. 2002; 26: 429-438.

14. Razvi R, Suri S, Sarma K, et al. Histomorphological and histochemical studies on the different layers of skin of Bakerwali goat. J Applied Animal Res. 2014; 43(2): 208-213, doi: 10.1080/09712119.2014.963089.

15. Sandby-Møller J, Poulsen T, Wulf HC. Epidermal thickness at different body sites: relationship to age, gender, pigmentation, blood content, skin type and smoking habits. Acta Derm Venereol. 2003; 83(6): 410-413, doi: 10.1080/00015550310015419, indexed in Pubmed: 14690333.

16. Sumena KB, Lucy KM, Chungath JJ, et al. Morphology of the skin in Large White Yorkshire pigs. Indian J Anim Res. 2010; 44: 55-57.

17. Saxena S, Malik M, Parekh H. Histological character of skin in crossbred cattle. IJVA. 1994; 6: 8-11.

18. Theerawatanasirikul S, SailasutaA, Suriyaphol G. Epidermal Thickness on Body Regions, Age, Sex and Breed in Normal Canine Skin: A Preliminary Study. 9th CU Veterinary Scientific Annual Conference. 2010.

19. Thomas JR. Effects of age and diet on rat skin histology. Laryngoscope. 2005; 115(3): 405-411, doi: $10.1097 / 01 . \mathrm{mlg} .0000157845 .86154 .48$, indexed in Pubmed: 15744148.

20. Treuting PM, Dintzis SM, Montine KS. Comparative Anatomy and Histology: A Mouse, Rat, and Human Atlas. Academic Press. 2017.

21. Wilson DE, Reeder DM. Mammal species of the world: a taxonomic and geographicreference. JHU Press. 2005.

22. Zaki SM. Characteristics of the skin of the female albino rats in different ages: histological, morphometric and electron microscopic Study. J Cytol Histol. 2015; s3, doi: 10.4172/2157-7099.s3-004.

23. Zanna G, Auriemma E, Arrighi S, et al. Dermoscopic evaluation of skin in healthy cats. Vet Dermatol. 2015; 26(1): 14-7, e3, doi: 10.1111/vde.12179, indexed in Pubmed: 25354768. 